\title{
Use of High-Frequency Jet Ventilation for Percutaneous Tumor Ablation
}

\author{
Alban Denys $\cdot$ Yann Lachenal $\cdot$ Rafael Duran • \\ Madeleine Chollet-Rivier $\cdot$ Pierre Bize
}

Received: 12 November 2012/Accepted: 7 December 2012/Published online: 2 May 2013

(C) Springer Science+Business Media New York and the Cardiovascular and Interventional Radiological Society of Europe (CIRSE) 2013

\begin{abstract}
Purpose To report feasibility and potential benefits of high-frequency jet ventilation (HFJV) in tumor ablations techniques in liver, kidney, and lung lesions.

Methods This prospective study included 51 patients (14 women, mean age 66 years) bearing 66 tumors (56 hepatic, 5 pulmonary, 5 renal tumors) with a median size of $16 \pm 8.7 \mathrm{~mm}$, referred for tumor ablation in an intentionto-treat fashion before preoperative anesthesiology visit. Cancellation and complications of HFJV were prospectively recorded. Anesthesia and procedure duration, as well as mean $\mathrm{CO}_{2}$ capnea, were recorded. When computed tomography guidance was used, 3D spacial coordinates of an anatomical target $<2 \mathrm{~mm}$ in diameter on 8 slabs of 4 slices of $3.75-\mathrm{mm}$ slice thickness were registered.

Results HFJV was used in 41 of 51 patients. Of the ten patients who were not candidate for HFJV, two patients had contraindication to HFJV (severe COPD), three had lesions invisible under HFJV requiring deep inspiration apnea for tumor targeting, and five patients could not have HFJV because of unavailability of a trained anesthetic team. No specific complication or hypercapnia related to HFJV were observed despite a mean anesthetic duration of $2 \mathrm{~h}$ and ventilation performed in procubitus $(n=4)$ or lateral decubitus $(n=6)$. Measured internal target
\end{abstract}

\footnotetext{
A. Denys $(\bowtie) \cdot$ Y. Lachenal · R. Duran · P. Bize

Department of Radiology and Interventional Radiology,

Lausanne University Hospital, 46 rue du Bugnon,

1011 Lausanne, Switzerland

e-mail: alban.denys@chuv.ch

M. Chollet-Rivier

Department of Anesthesiology, Lausanne University Hospital,

46 rue du Bugnon, 1011 Lausanne, Switzerland
}

movement was $0.3 \mathrm{~mm}$ in $x$ - and $y$-axis and below the slice thickness of $3.75 \mathrm{~mm}$ in the $z$-axis in 11 patients.

Conclusions HFJV is feasible in $80 \%$ of patients allowing for near immobility of internal organs during liver, kidney, and lung tumor ablation.

Keywords Ablation - Anestheia - Jet-ventilation . Computed tomography

$\begin{array}{ll}\text { Abbreviations } \\ \text { RFA } & \text { Radiofrequency ablation } \\ \text { MWA } & \text { Microwave ablation } \\ \text { CT } & \text { Computed tomography } \\ \text { US } & \text { Ultrasound } \\ \text { HFJV } & \text { High-frequency jet ventilation }\end{array}$

Percutaneous thermal ablative techniques, such as radiofrequency ablation (RFA), microwave ablation (MWA), or cryoablation, have become valuable alternatives to surgery in selected patients with liver, kidney, or lung malignancies [1-4]. During the procedure, one or several thermal probes are placed in the target lesion under computed tomography (CT) or ultrasound (US) guidance and thermal energy is deposited in the tissue to achieve tissue destruction. The success of this treatment modality is directly related to the ability to induce both destruction of the tumor and of a circumferential rim of normal parenchyma around the tumor [5]. Taking into consideration that most devices are able to create a single ablation of $\sim 4 \mathrm{~cm}$ in diameter [6] and that, for instance, RFA or MWA is proposed for liver lesions up to $3 \mathrm{~cm}$ in diameter, a precise positioning of probes in the center of the lesion is critical. Quality and reliability of the imaging guidance as well as operator's experience is therefore capital. Another parameter that 
needs to be taken into account is the mobility of the organs during needle placement. Until recently, there was no other way to obtain immobility of targeted lesions located in the liver, kidney, or lung than apnea for patients under general anesthesia.

High-frequency jet ventilation (HFJV) is a mechanical ventilation method where high-flow, short-duration pulses of pressurized gaz are delivered in the trachea through a small caliber catheter [7]. Gas exchange is assured by highvelocity gas flow washing the alveoli [8]. Initially, HFJV has been developed for specific indications, such as surgery of the larynx and trachea, because direct placement of this HFJV catheter can be done though a minitracheostomy leaving the larynx and trachea fully accessible for surgery. Because HFJV is made of short and small pulses of air, it induces very small amplitude respiratory movements. This might be of significant interest for percutaneous ablation procedures as recently demonstrated for other technics, such as extracorporeal shockwave lithotripsy for kidney stones $[9,10]$ and lung tumor radiotherapy [11]. However, HFJV has its own limitations and carries a higher risk of barotraumatic pneumothorax; its use is limited by some contraindications, such as severe chronic obstructive pulmonary disease, obesity, recent pneumothorax, and recent pulmonary surgery [12]. Furthermore, the diaphragm and inner organs are maintained in an intermediate position between inspiration and expiration, which might sometimes limit access to high located lesions in the liver or kidney.

A recent pilot study has suggested that HFJV could be used for the tumor ablation [13]. This study described the use of HFJV in nine patients. This small number of cases did not demonstrate absence of organ movements during this method of ventilation and did not illustrate risk or pitfalls of this technique in this particular indication. Thus, our study was designed to document the use of HFJV in a larger subset of patients treated by tumor ablation, to measure organ movements during HFJV, and to illustrate benefit and limits of this technique.

\section{Materials and Methods}

This prospective, single-center study was conducted in a university hospital and was approved with a waiver of informed consent by the institutional review board of the same hospital.

\section{Study Design}

Patients selected for local ablation after tumor board meeting decision and after preoperative consultation with interventional radiologists between May and December
2011 were included in intention to treat before the preoperative anesthesiologic visit. Inclusion criteria for the study were age older than 18 years, informed consent of the study obtained, and lesion accessible to percutaneous treatment.

A total of 51 patients were identified (Table 1). No patients were excluded based on our criteria; thus, the final study population included 51 patients [14 women and 37 men; mean age 66 (range 37-80) years].

A total of 66 lesions were identified. Fifty-six lesions were in the liver (30 hepatocellular carcinomas, 1 intrahepatic cholangiocarcinoma, 25 liver metastases), 5 in the kidneys (4 renal cell carcinomas, one oncocytoma) and in the lungs ( 2 epidermoid carcinomas, 3 lung metastases). Lesions were solitary in 37 patients $(73 \%)$ and multiple in $14(27 \%)$; there were 2 lesions in 13 patients, and 3 lesions in 1 patient.

\section{Anesthesia Protocol}

All patients had a preanesthesia visit and were classified according to the physical status classification system of the American Society of Anesthesiologists (ASA). Informed consent for anesthesia was obtained. During this visit, all contraindications for HFJV were specifically searched and recorded when present. HFJV was contraindicated in case of severe COPD (VEMS $<1,500 \mathrm{ml} / \mathrm{s}$ ), recent pneumothorax or recent thoracic surgery because of the risk of baro-traumatism, or severe obesity because of the risk of hypercapnia [8].

After induction, anesthesia was maintained by a total intravenous technique with propofol $(0.15-0.2 \mathrm{mg} / \mathrm{kg} / \mathrm{min})$ and remifentanil $(0.2-0.3 \mu \mathrm{g} / \mathrm{kg} / \mathrm{min})$. Orotracheal intubation was performed under curare (vecuronium $0.1 \mathrm{mg} / \mathrm{kg}$ ) and conventional mechanical ventilation initiated. Just before imaging, the anesthesia circuit was disconnected, a dedicated 2-mm diameter jet catheter (Acutronic Medical System $\mathrm{AG}^{\circledR}$, Hirzel, Switzerland) was slipped into the endotracheal tube (Figs. 1, 2) and automatic HFJV was initiated at a frequency of $150-250 / \mathrm{min}$ and a driving pressure of 1-2 bar using the Monsoon jet ventilator (Acutronic Medical System $\mathrm{AG}^{\circledR}$ ). Standard monitoring consisted of blood pressure, ECG, peripheral $\mathrm{O}_{2}$ saturation, ETCO2 during conventional mechanical ventilation and bispectral index BIS (Philips Intellivue MP50 ${ }^{\circledR}$, Switzerland). Specific monitoring during HFJV consisted of transcutaneous $\mathrm{CO}_{2}$ (PTO2/PTCO2 Tosca TCM $4{ }^{\circledR}$, Radiometer GMBH, Switzerland), alarm pressure cutoff of the jet ventilator, as well as clinical monitoring of chest movements and expiratory drive. At the end of the percutaneous thermal ablation, the HFJV catheter was removed, conventional ventilation was resumed via the anesthetic circuit and patient was awakened and extubated. 
Table 1 Flowchart of patient

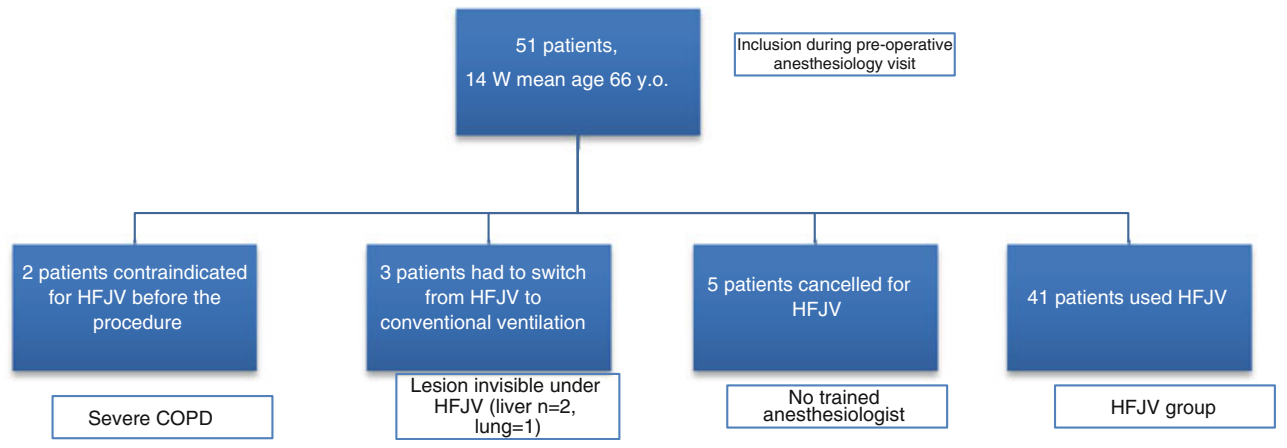

As HFJV carries a higher risk of hypercapnia motivating a switch to conventional high-pressure ventilation [12], mean capnea was monitored as well as any anesthesiarelated complication. Duration of anesthesia was registered.

\section{Imaging Protocol}

An interventional suite dedicated to percutaneous procedures including a 128-multidetector CT (Phillips Ingenuity, Best, The Netherlands), an US unit (IU22 Phillips Healthcare, Best, The Netherlands) and the PercuNAV image fusion and navigation system (PercuNAV, Best, The Netherlands) were used.

CT was used in 11 patients as a guiding method for needle placement in 6 thoracic tumors and in 7 liver tumors invisible or poorly delineated under ultrasound. Acquisition were obtained on the targeted organ after injection of iodinated nonionic contrast agent in all patients $(300 \mathrm{mg}$ of iodine per $\mathrm{ml}$ ) at a flow rate of $3 \mathrm{ml} / \mathrm{sec}$ (volume in milliliters = body weight $+30 \mathrm{ml}$ ) either at the arterial (20-25 s) and venous phase (60-70 s) for abdominal

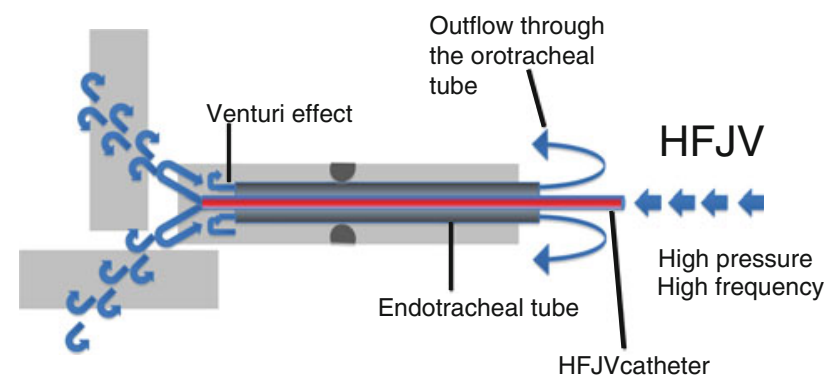

Air turbulences caused by the

high velocity flow maintain

correct oxygenation at the

alveolar level

Fig. 1 Schematic drawing showing HFJV mechanism and set-up in the trachea and bronchi. Small pushes of gaz injected at high pressure in the HFJV catheter are pushed one by each other from the trachea to the bronchus to allow oxygenation. The endotracheal tube is left opened to allow gaz outflow organs or at the venous phase (60-70 s) for thoracic procedure. After having chosen the reference plan for needle placement, repeated acquisitions at this level were obtained with a slab of 4 slices of $3.75-\mathrm{mm}$ thickness $(120 \mathrm{kV}$, $300 \mathrm{~mA}$, table speed of $55 \mathrm{~mm}$ per rotation [0.7 s], pitch of 1.375). Dose length product was registered for each procedure (mGy/cm).

For organ movement measurement, a precise landmark in the targeted organ, such as a vascular calcification, a vascular, or a biliary bifurcation, was chosen on a central image of the 4 slices acquisition. This landmark was chosen to be distant of the needle tract or tumor to avoid displacement due to needle positioning. Spatial coordinates of this landmark were registered on 8 consecutive slabs in $x$-, $y$-, and $z$-axis. For $z$-axis monitoring, our measurement accuracy was limited to slice thickness or multiple of it $(3.75,7.5 \mathrm{~mm}$, and so on). Therefore, the lower limit of movement measurement in the $z$ direction is $3.75 \mathrm{~mm}$.

For liver and kidney tumors, we used ultrasound guidance using a 3.5-Mhz, curved, array probe, and we used harmonic imaging at operator's discretion. When lesions were not visible under US and transiently identified after injection of contrast medium on CT, we used the PercuNAV navigation system. This system allowed real time fusion of preacquired CT images and US pending cutaneous and internal anatomic landmarks coregistration [14].

\section{Procedure}

Once the target lesion was identified either with US or CT, the maximal diameter of the lesion as well as the distance of the lesion to the skin entry point of the ablation probe was noted in the study database. The ablation probes were then advanced to their final position under HFJV using either CT or/and US guidance. When necessary, correction of the needle position was performed. The final position of the ablation applicators was confirmed by a spiral CT acquisition before the ablation was initiated. For all 

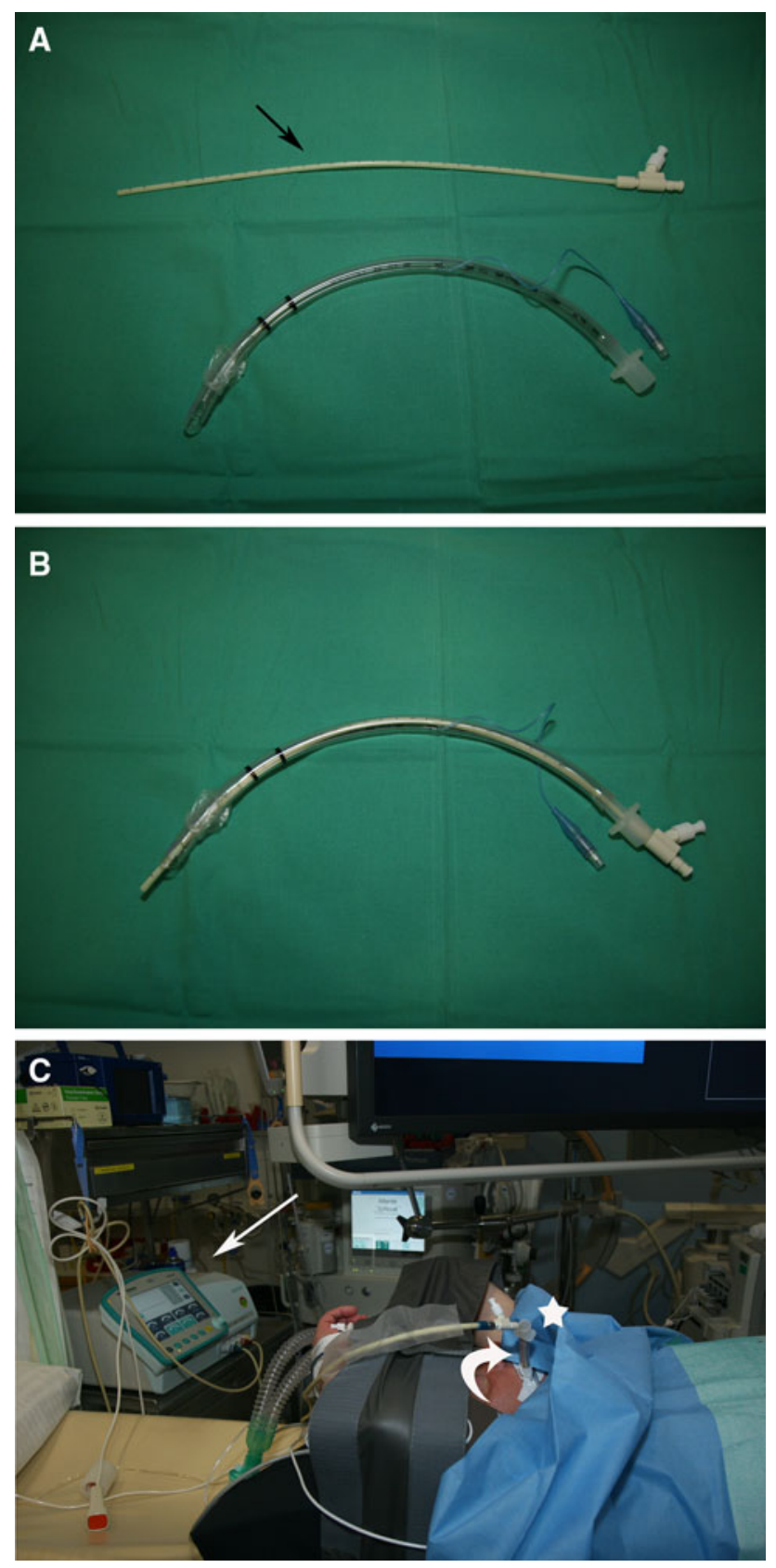

Fig. 2 A The HFJV cannula is a long straight single lumen tube of $2 \mathrm{~mm}$ in diameter (black arrow). B During the procedure, it is inserted into the orotracheal tube, which is left open to allow gaz expulsion. C During the procedure, the small cannula (curved arrow) coming from the Mansoon ventilation machine (white arrow) is inserted in the orotracheal cannula (white arrow), which is left open

patients $\mathrm{CT}$ acquisition was done at the end of the procedure to evaluate extent of ablation, for possible complication depiction (pneumothorax, bleeding) and, for 11 patient who had a CT as guiding method for needle placement, to measure organ movement during HFJV. Additional thermal ablations were performed when needed. All images acquisitions were performed under HFJV.

\section{Ablation Protocols}

For RFA, internally cooled radiofrequency probe (Cluster needle E series, Covidien, Dublin, Ireland) or coaxial radiofrequency ablation device (LeVeen CoAccess ${ }^{\mathrm{TM}}$ Electrode System, Boston Scientific, Natick, MA) were used. For MWA, microwave antennas (Accu 2i pMTA Microsulis, Hampstead, United Kingdom) and for cryoablation, cryoprobes (Galil Medical, Yokneam, Israel) were used. Liver lesions were treated by RFA or MWA depending on their location and size. Renal tumors were treated by RFA when they were exophytic and of small size, whereas larger lesions (superior to $3 \mathrm{~cm}$ in diameter) or lesions positioned close to the renal hilum or ureter were treated by cryoablation. All primary or secondary pulmonary tumors were treated by RFA. Standard ablation protocols recommended by companies were used. RFA ablation cycle lasted $12 \mathrm{~min}$ using Covidien perfused needles and was depending on impedance roll-off phenomenon for Boston Scientific multiprong needles, whereas MWA lasted $6 \mathrm{~min}$ at $180 \mathrm{~W}$ and cryoablation lasted twice 20 min (each 20 min cycle includes freezing, passive, and active thawing). Duration of the ablative procedure was registered.

Complications during and after treatment were registered using the Society of Interventional Radiology classification [15].

\section{Results}

Anesthesia Protocol

Physical status of patients was II in 30 patients and III in 21 patients according to ASA score. HFJV was used in 41 out of 51 patients. Two patients had severe COPD grade 2 and could not be ventilated using this technique. In 3 patients, HFJV made lesion either inaccessible $(n=1$, pulmonary metastasis from melanoma) or invisible ( $n=2$, small liver metastasis in segment 8 ). We had to switch to conventional ventilation method and to use apnea under high positive pressure ventilation to make lesions accessible/visible under US guidance. In one patient having lung metastases in the posterior part of the lung, the lesion was obscured by atelectasia under HFJV, which resolved under conventional positive pressure ventilation. In five cases, HFJV was cancelled due to the absence of anesthesiologist certified for this technique and patients received conventional positive pressure ventilation. When treatment procedure necessitated a different position than decubitus, HFJV also could be used in patients positioned in procubitus $(n=4)$ or in lateral position $(n=6)$. 
Mean duration of anesthesia from induction to awakening was $2 \mathrm{~h}$ (range: $1 \mathrm{~h}$ and $4 \mathrm{~min}-2 \mathrm{~h}$ and $55 \mathrm{~min}$ ), with a mean procedure duration of $1 \mathrm{~h}$ and $17 \mathrm{~min}$ (range, $35 \mathrm{~min}-2 \mathrm{~h}$ and $15 \mathrm{~min}$ ). Duration of anesthesia in patients who received HFJV compared with conventional ventilation was slightly longer, $2 \mathrm{~h}$ and $5 \mathrm{~min}$ versus $1 \mathrm{~h}$ and 43 min respectively. The mean $\mathrm{PCO}_{2}$ was $37 \mathrm{mmHg}$ in the series with no difference between HFJV and conventional ventilation patients (respectively $37 \pm 6.5$, and $36 \pm 3.7 \mathrm{mmhg}$ ). We had 10 patients with per anesthesia complications (2 delayed awakening, 2 hypotension, 1 difficult intubation, 1 extubation during procedure, 4 spontaneously resolutive cardiac arrhythmias) and one severe postanesthesia complication consisting in a brachial plexus paralysis due to arm mal positioning during the procedure.

\section{Ablative Procedures}

RFA was used in 41 patients, MWA in 7 and cryoablation in 3 patients. The mean size of the treated lesion was $1.8 \pm 0.87 \mathrm{~cm}$ (range, $0.7-4 \mathrm{~cm}$ ) and the skin to tumor distance was $10 \pm 3.1 \mathrm{~cm}$ (range, 4-16.2 cm). For small hardly visible lesions under US, PercuNAV was used in 5 patients bearing 9 lesions ( 5 liver metastasis, 3 hepatocellular carcinoma). These lesions were smaller than the overall population (mean $1.2 \pm 0.4 \mathrm{~cm}$ ) and more deeply located for ablation (mean skin to tumor distance: $11.7 \pm 2.4 \mathrm{~cm})$. Isolation from adjacent organs using hydrodissection was used in 3 patients (colon, $n=2$ and stomach, $n=1$ ). Percutaneous balloon occlusion of hepatic veins was used in 2 patients [16]. One patient had a right portal vein embolization during the same procedure. Three patients necessitated repeated ablation after control CT performed at the end of the procedure due to insufficient safety margin around the ablated lesion (two liver metastases and one renal cell carcinoma). During and in the 30 days after the procedure, 18 of 51 patients experienced complications according to SIR classification (Type A $n=10$, Type $\mathrm{B} n=2$, Type $\mathrm{C} n=1$, Type $\mathrm{D} n=4$, Type $\mathrm{E} n=1$; Table 2). None of these complications could be attributed to the ventilation method. Mean DLP for the procedure was $1,177.5 \mathrm{mGy} / \mathrm{cm}$ with no difference between patients under HFJV (mean 1,192.36 mGy/cm) or conventional ventilation $(1,116.57 \mathrm{mGy} / \mathrm{cm})$.

\section{Organ Movement During HFJV}

Organ movement was measured in 11 patients (9 liver, 2 lung) using 8 repeated slabs of 4 slices of $3.75 \mathrm{~mm}$ in slice thickness during needle positioning. During HFJV, the mean movement of target is $0.3 \pm 0.12,0.3 \pm 0.13$, and under $3.75 \mathrm{~mm}$ in $x, y$, and $z$ directions respectively
Table 2 Complications related to the procedure according to SIR classification [21]

\begin{tabular}{ll}
\hline A & $n=4$ \\
Pneumothorax not drained & $n=5$ \\
Perihepatic hematoma asymptomatic & $n=1$ \\
Moderate fever at $48 \mathrm{~h}$ & $n=1$ \\
Parietal hematoma & $n=1$ \\
B & \\
$\quad$ Acute pain during 72 h & $n=1$ \\
C & \\
Chronic abdominal pain lasting one month & $n=1$ \\
D & $n=1$ \\
Colic perforation during needle placement & $n=1$ \\
Sepsis at day 2 & $n=1$ \\
Hemobilia during ablation embolized & \\
$\quad$ Delayed cholangitis at 1 month & $n=1$ \\
E & \\
Brachial plexus lesion during anesthesia &
\end{tabular}

(mean $\pm \mathrm{SD}$ ). Range of movement of targets is $0-1.06$, $0.1-1.14$, and $<3.75 \mathrm{~mm}$ in $x$-, $y$-, and $z$-axis respectively (Fig. 3).

\section{Discussion}

Our study demonstrated that HFJV is a useful anesthetic ventilation method during percutaneous ablation procedures. In our study group, this method was perfectly applicable for different ablation methods in various organs including liver, kidney, and lung. HFJV can be used as demonstrated in our study even if the patient is positioned in procubitus or lateral decubitus. HFJV could be used for long procedures, such as combination of portal vein embolization and RFA lasting $2 \mathrm{~h}$. In our series, pending strict selection criteria, no patient experienced hypercapnia during anesthesia, and HFJV had never been stopped because of ventilation difficulties.

Our study demonstrated than organ movement in $x$ - and $y$-axis was extremely limited (below $1 \mathrm{~mm}$ ) in $x$ - and $y$-axis. Because slice thickness was $3.75 \mathrm{~mm}$, we could only measure target movement to be below $3.75 \mathrm{~mm}$. However, everyone will admit that this always less than the standard liver vertical movement observed during breathing or ventilation. Using conventional ventilation during general anesthesia, conventional ventilator settings are usually adjusted to deliver between 6 and $10 \mathrm{cc} / \mathrm{kg}$ of tidal volume. A tidal volume of $600-800 \mathrm{cc}$ is always associated with movements of lungs, chest wall, and abdominal organs [7]. During normal breathing, which uses similar tidal volumes, organ motion was studied for purpose of lung and abdominal radiotherapy. Movement amplitude in 

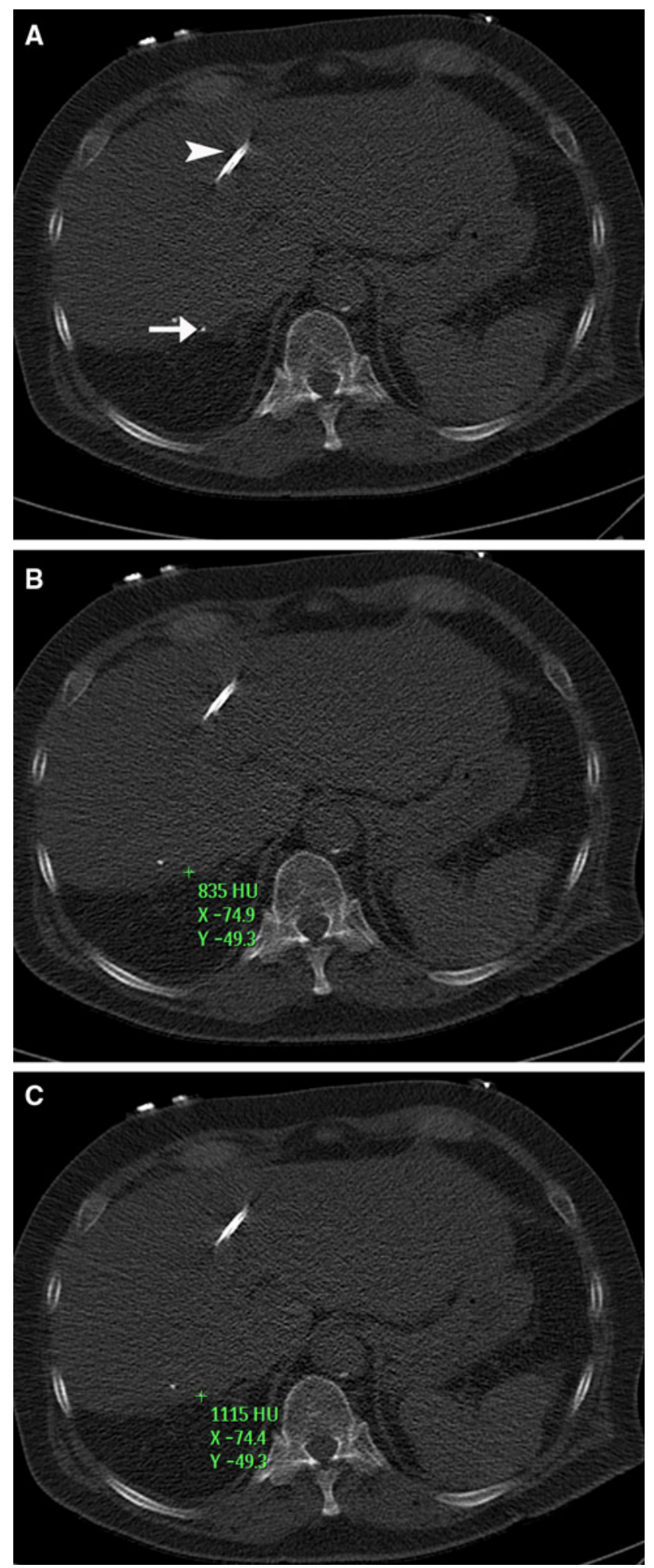

Fig. 3 Repeated CT acquisitions at the same level during needle placement (white arrow head) in a segment 8 liver metastasis. A A punctiform calcification is identified in segment 7 (bold white arrow). $\mathbf{B}$, C Spatial coordinates in $\mathrm{x}$ - and $\mathrm{y}$-directions of this calcification shows no significant movement during HFJV the vertical direction has been described to be between 1 and $2 \mathrm{~cm}$ [17]. To our knowledge, our study is the first in the literature to evaluate organ movement during HFJV in human. HFJV has already demonstrated some clinical benefits in non randomized trial for atrial fibrillation ablation with shorter procedure duration [18] and for extracorporeal shock wave lithotripsy with lower number of shocks and less energy [19]. In a pilot retrospective study, Abderhalden et al. [13] found that HFJV reduced the dose-length product delivered to the patient compared with conventional ventilation in a series of nine patients receiving liver or kidney ablation during HFJV. We did not observe this difference in our studies.

This method seems perfectly adapted for more advanced guiding techniques, such as real-time fusion imaging. In this technique, CT images are fused in real time with US acquisition allowing for superposition on US images of tumors only visible during CT acquisition after contrast media injection [14]. Using this method, we were able to treat deeply located tumors invisible under US with an excellent precision using PercuNAV. HFJV also opens the gate for more advanced technique, such as robotic placement of needles, or precise treatment planning in large tumors where multiple overlapping ablations and/or multiple applicators are needed. One indication of HFJV is high-intensity, ultrasound ablation method to improve the $5 \%$ rate of major complications in liver tumor ablation and $8 \%$ in pancreatic tumor ablation [20]. Jung et al. [20] emphasized the necessity of precise targeting of the treated area and the risk of adjacent organ complication (myocardium, spine, duodenum). Immobility of the target lesion might help to reduce this complication rate.

HFJV has some limitations. It has its own contraindications as recommended by Bourgain et al. [8], such as COPD, recent pneumothorax, or severe obesity. These contraindications are not absolute and the risk of the anesthesia could be weighted by the potential benefit of the procedure. HFJV is not widely used therefore not all anesthesiologist are confident in using it. In our study $10 \%$ of cases could not be treated using HFJV because anesthesiologist team was not trained. More extensive use of this method should avoid these problems in the future. Another potential advantage of this technique lies in the lower peak airway pressures used. This could be an advantage in the case of lung tumor ablation as we can expect that air-leaks though the pleura should be limited in comparison with ablation performed under conventional positive airway pressure, which use much higher pressure.

In three patients $(6 \%)$, HFJV made tumor ablation impossible because it made lesion either inaccessible or invisible. Indeed patients under HFJV had the diaphragm in 
intermediate position because intra-alveolar and intrapulmonary pressure are intermediate, which may make some lesions (e.g. in the dome of the liver) difficult to access or make lung lesion invisible due to atelectasis. For these three patients, HFJV could be switched to conventional ventilation with apneas under positive pressure ventilation, which made the lesions visible, and ablations could be performed.

In conclusion, we found the use of HFJV very useful for the ablation of tumors in the liver, kidneys, and lungs. Our study demonstrated barely any organ movement during ventilation. Furthermore, the risk of complications related to this technique seems limited pending a trained anesthetic team. This ventilation technique opens the gate for more sophisticated development, such as robotic, and the use of ablation techniques necessitating long ablation cycles with external energy sources, such as high-intensity focused ultrasound. In our study, we did not use CT fluoroscopy, which also is a useful tool for difficult placement and might have reduced procedure time.

Conflict of Interest Alban Denys has COI to disclose (consultancy, Terumo; grants: Terumo, biocompatible; lectures: Terumo) none of the other authors have any conflict of interest related or unrelated to the study to disclose.

\section{References}

1. Cutress ML, Ratan HL, Williams ST, O’Brien MF (2010) Update on the management of $\mathrm{T} 1$ renal cortical tumours. BJU Int 106(8):1130-1136

2. Guenette JP, Dupuy DE (2010) Radiofrequency ablation of colorectal hepatic metastases. J Surg Oncol 102(8):978-987

3. Rempp H, Boss A, Helmberger T, Pereira P (2011) The current role of minimally invasive therapies in the management of liver tumors. Abdom Imaging 36(6):635-647

4. Sonntag PD, Hinshaw JL, Lubner MG, Brace CL, Lee FT Jr (2011) Thermal ablation of lung tumors. Surg Oncol Clin N Am 20(2):369-387 ix

5. Wong SL, Mangu PB, Choti MA et al (2010) American Society of Clinical Oncology 2009 clinical evidence review on radiofrequency ablation of hepatic metastases from colorectal cancer. J Clin Oncol 28(3):493-508

6. de Baere T, Denys A, Wood BJ et al (2001) Radiofrequency liver ablation: experimental comparative study of water-cooled versus expandable systems. AJR Am J Roentgenol 176(1):187-192
7. Raiten J, Elkassabany N, Mandel JE (2012) The use of highfrequency jet ventilation for out of operating room anesthesia. Curr Opin Anaesthesiol 25(4):482-485

8. Bourgain JL, Chollet M, Fischler M, Gueret G, Mayne A (2010) Guide for the use of jet-ventilation during ENT and oral surgery. Ann Fr Anesth Reanim 29(10):720-727

9. Cormack JR, Hui R, Olive D, Said S (2007) Comparison of two ventilation techniques during general anesthesia for extracorporeal shock wave lithotripsy: high-frequency jet ventilation versus spontaneous ventilation with a laryngeal mask airway. Urology 70(1):7-10

10. Olive DJ, Cormack JR (2006) Kidney stone movement during lithotripsy under general anaesthesia: high frequency jet ventilation versus spontaneous ventilation. Anaesth Intensive Care 34(6):832-833

11. Fritz P, Kraus HJ, Muhlnickel W, Sassmann V, Hering W, Strauch K (2010) High-frequency jet ventilation for complete target immobilization and reduction of planning target volume in stereotactic high single-dose irradiation of stage I non-small cell lung cancer and lung metastases. Int J Radiat Oncol Biol Phys 78(1):136-142

12. Fernandez-Bustamante A, Ibanez V, Alfaro JJ et al (2006) Highfrequency jet ventilation in interventional bronchoscopy: factors with predictive value on high-frequency jet ventilation complications. J Clin Anesth 18(5):349-356

13. Abderhalden S, Biro P, Hechelhammer L, Pfiffner R, Pfammatter $\mathrm{T}$ (2011) CT-guided navigation of percutaneous hepatic and renal radiofrequency ablation under high-frequency jet ventilation: feasibility study. J Vasc Interv Radiol 22(9):1275-1278

14. Abi-Jaoudeh N, Kruecker J, Kadoury S et al (2012) Multimodality image fusion-guided procedures: technique, accuracy, and applications. Cardiovasc Intervent Radiol 35(5):986-998

15. Sacks D, McClenny TE, Cardella JF, Lewis CA (2003) Society of Interventional Radiology clinical practice guidelines. J Vasc Interv Radiol 14(9 Pt 2):S199-S202

16. de Baere T, Bessoud B, Dromain C et al (2002) Percutaneous radiofrequency ablation of hepatic tumors during temporary venous occlusion. AJR Am J Roentgenol 178(1):53-59

17. Langen KM, Jones DT (2001) Organ motion and its management. Int J Radiat Oncol Biol Phys 50(1):265-278

18. Goode JS Jr, Taylor RL, Buffington CW, Klain MM, Schwartzman D (2006) High-frequency jet ventilation: utility in posterior left atrial catheter ablation. Heart Rhythm 3(1):13-19

19. Mucksavage P, Mayer WA, Mandel JE, Van Arsdalen KN (2010) High-frequency jet ventilation is beneficial during shock wave lithotripsy utilizing a newer unit with a narrower focal zone. Can Urol Assoc J 4(5):333-335

20. Jung SE, Cho SH, Jang JH, Han JY (2011) High-intensity focused ultrasound ablation in hepatic and pancreatic cancer: complications. Abdom Imaging 36(2):185-195

21. Sacks D, McClenny TE, Cardella JF, Lewis CA (2003) Society of Interventional Radiology clinical practice guidelines. J Vasc Interv Radiol 14(9 Pt 2):S199-S202 